\title{
Iterative Algorithms with Perturbations for Solving the Systems of Generalized Equilibrium Problems and the Fixed Point Problems of Two Quasi-Nonexpansive Mappings
}

\author{
Rabian Wangkeeree and Uraiwan Boonkong \\ Department of Mathematics, Faculty of Science, Naresuan University, Phitsanulok 65000, Thailand
}

Correspondence should be addressed to Rabian Wangkeeree, rabianw@nu.ac.th

Received 3 September 2012; Accepted 1 November 2012

Academic Editor: Xiaolong Qin

Copyright (C) 2012 R. Wangkeeree and U. Boonkong. This is an open access article distributed under the Creative Commons Attribution License, which permits unrestricted use, distribution, and reproduction in any medium, provided the original work is properly cited.

\begin{abstract}
We introduce new iterative algorithms with perturbations for finding a common element of the set of solutions of the system of generalized equilibrium problems and the set of common fixed points of two quasi-nonexpansive mappings in a Hilbert space. Under suitable conditions, strong convergence theorems are obtained. Furthermore, we also consider the iterative algorithms with perturbations for finding a common element of the solution set of the systems of generalized equilibrium problems and the common fixed point set of the super hybrid mappings in Hilbert spaces.
\end{abstract}

\section{Introduction}

Let $H$ be a real Hilbert space with inner product $\langle\cdot, \cdot\rangle$ and norm $\|\cdot\|$ and $C$ a nonempty closed convex subset of $H$ and let $T$ be a mapping of $C$ into $H$. Then, $T: C \rightarrow H$ is said to be nonexpansive if $\|T x-T y\| \leq\|x-y\|$ for all $x, y \in C$. A mapping $T: C \rightarrow H$ is said to be quasinonexpansive if $\|T x-y\| \leq\|x-y\|$ for all $x \in C$ and $y \in F(T):=\{x \in C: T x=x\}$. It is well known that the set $F(T)$ of fixed points of a quasi-nonexpansive mapping $T$ is closed and convex; see Itoh and Takahashi [1]. A mapping $T: C \rightarrow H$ is called nonspreading [2] if

$$
2\|T x-T y\|^{2} \leq\|T x-y\|^{2}+\|T y-x\|^{2},
$$

for all $x, y \in C$. We remark that nonlinear every nonspreading mappings are quasi-nonexpansive mappings if the set of fixed points is nonempty. 
Recall that a mapping $\Psi: C \rightarrow H$ is said to be $\mu$-inverse strongly monotone if there exists a positive real number $\mu$ such that

$$
\langle\Psi x-\Psi y, x-y\rangle \geq \mu\|\Psi x-\Psi y\|^{2}, \quad \forall x, y \in C .
$$

If $\Psi$ is a $\mu$-inverse strongly monotone mapping of $C$ into $H$, then it is obvious that $\Psi$ is $1 / \mu$ Lipschitz continuous.

Let $G: C \times C \rightarrow \mathbb{R}$ be a bifuction and $\Psi: C \rightarrow H$ be $\mu$-inverse strongly monotone mapping. The generalized equilibrium problem (for short, GEP) for $F$ and $\Psi$ is to find $z \in C$ such that

$$
G(z, y)+\langle\Psi z, y-z\rangle \geq 0, \quad \forall y \in C
$$

The problem (1.3) was studied by Moudafi [3]. The set of solutions for problem (1.3) is denoted by $\operatorname{GEP}(F, \Psi)$, that is,

$$
\operatorname{GEP}(F, \Psi)=\{z \in C: G(z, y)+\langle\Psi z, y-z\rangle \geq 0, \forall y \in C\}
$$

If $\Psi \equiv 0$ in (1.3), then GEP reduces to the classical equilibrium problem and $\operatorname{GEP}(G, 0)$ is denoted by $\operatorname{EP}(G)$, that is,

$$
\operatorname{EP}(G)=\{z \in C: G(z, y) \geq 0, \forall y \in C\}
$$

If $G \equiv 0$ in (1.3), then GEP reduces to the classical variational inequality and $\operatorname{GEP}(0, \Psi)$ is denoted by $\operatorname{VI}(\Psi, C)$, that is,

$$
\mathrm{VI}(\Psi, C)=\{z \in C:\langle\Psi z, y-z\rangle \geq 0, \forall y \in C\} .
$$

The problem (1.3) is very general in the sense that it includes, as special cases, optimization problems, variational inequalities, Min-Max problems, the Nash equilibrium problems in noncooperative games, and others; see, for example, Blum and Oettli [4] and Moudafi [3].

In 2005, Combettes and Hirstoaga [5] introduced an iterative algorithm of finding the best approximation to the initial data and proved a strong convergence theorem. In 2007, by using the viscosity approximation method, S. Takahashi and W. Takahashi [6] introduced another iterative scheme for finding a common element of the set of solutions of the equilibrium problem and the set of fixed points of a nonexpansive mapping. Subsequently, algorithms constructed for solving the equilibrium problems and fixed point problems have further developed by some authors. In particular, Ceng and Yao [7] introduced an iterative scheme for finding a common element of the set of solutions of the mixed equilibrium problem and the set of common fixed points of finitely many nonexpansive mappings. Mainge and Moudafi [8] introduced an iterative algorithm for equilibrium problems and fixed point problems. Wangkeeree [9] introduced a new iterative scheme for finding the common element of the set of common fixed points of nonexpansive mappings, the set of solutions of an equilibrium problem, and the set of solutions of the variational inequality. Wangkeeree and Kamraksa [10] introduced an iterative algorithm for finding a common element of the set of solutions of a mixed equilibrium problem, the set of fixed points of an infinite family of 
nonexpansive mappings and the set of solutions of a general system of variational inequalities for a cocoercive mapping in a real Hilbert space. Their results extend and improve many results in the literature.

In 1967, Wittmann [11] (see also [12]) proved the strong convergence theorem of Halpern's type [13] $\left\{x_{n}\right\}$ defined by, for any $x_{1}=x \in C$,

$$
x_{n+1}=\alpha_{n} x+\left(1-\alpha_{n}\right) T x_{n}, \quad \forall n \in \mathbb{N},
$$

where $\left\{\alpha_{n}\right\} \subset(0,1)$ satisfies $\lim _{n \rightarrow \infty} \alpha_{n}=0, \sum_{n=1}^{\infty} \alpha_{n}=\infty$, and $\sum_{n=1}^{\infty}\left|\alpha_{n}-\alpha_{n+1}\right|<\infty$. In [14], Kurokawa and Takahashi also studied the following Halpern's type for nonspreading mappings in a Hilbert space; see also Hojo and Takahashi [15]. Let $T$ be a nonspreading mapping of $C$ into itself. Let $u \in C$ and define two sequences $\left\{x_{n}\right\}$ and $\left\{z_{n}\right\}$ in $C$ as follows: $x_{1}=$ $x \in C$ and

$$
x_{n+1}=\alpha_{n} u+\left(1-\alpha_{n}\right) z_{n}, \quad \text { where } z_{n}=\frac{1}{n} \sum_{k=0}^{n-1} T^{k} x_{n}
$$

for all $n=1,2, \ldots$, where $\left\{\alpha_{n}\right\} \subset(0,1), \lim _{n \rightarrow \infty} \alpha_{n}=0$ and $\sum_{n=1}^{\infty} \alpha_{n}=\infty$. If $F(T)$ is nonempty, they proved that $\left\{x_{n}\right\}$ and $\left\{z_{n}\right\}$ converge strongly to $P_{F(T)} u$, where $P_{F(T)}$ is the metric projection of $H$ onto $F(T)$. Recently, Yao and Shahzad [16] gave the following iteration process for nonexpansive mappings with perturbation: $x_{1} \in C$ and

$$
x_{n+1}=\left(1-\beta_{n}\right) x_{n}+\beta_{n} P_{C}\left(\alpha_{n} u_{n}+\left(1-\alpha_{n}\right) T x_{n}\right), \quad \forall n \in \mathbb{N},
$$

where $\left\{\alpha_{n}\right\}$ and $\left\{\beta_{n}\right\}$ are sequences in $[0,1]$, and the sequence $\left\{u_{n}\right\} \subseteq H$ is a small perturbation for the $n$-step iteration satisfying $\left\|u_{n}\right\| \rightarrow 0$ as $n \rightarrow \infty$. In fact, there are perturbations always occurring in the iterative processes because the manipulations are inaccurate.

On the other hand, very recently, Chuang et al. [17] considered the following iteration process for finding a common element of the set of solutions of the equilibrium problem and the set of common fixed points for a quasi-nonexpansive mapping $T: C \rightarrow H$ with perturbation

$$
q_{1} \in H,
$$

$$
\begin{gathered}
x_{n} \in C, \quad \text { such that } G\left(x_{n}, y\right)+\frac{1}{r_{n}}\left\langle y-x_{n}, x_{n}-q_{n}\right\rangle \geq 0, \quad \forall y \in C, \\
y_{n}=\beta_{n} x_{n}+\left(1-\beta_{n}\right) T x_{n}, \\
q_{n+1}=\alpha_{n} u_{n}+\left(1-\alpha_{n}\right) y_{n}, \quad \forall n \in \mathbb{N},
\end{gathered}
$$

where $C$ is a nonempty closed convex subset of $H, G: C \times C \rightarrow \mathbb{R}$ is a function, $\left\{\alpha_{n}\right\}$ and $\left\{\beta_{n}\right\}$ are real sequences in $(0,1)$, and $\left\{u_{n}\right\} \subset H$ is a convergent sequence and $\left\{r_{n}\right\} \subset[a, \infty)$ for some $a>0$. They obtained a strong convergence theorem for such iterations.

In this paper, inspired and motivated by Yao and Shahzad [16], S. Takahashi and W. Takahashi [18] and Chuang et al. [17], we introduce a new iterative algorithms with perturbations for finding a common element of the set of solutions of the system of generalized equilibrium problems and the set of common fixed points of two quasi-nonexpansive 
mappings in a Hilbert space. Under suitable conditions, strong convergence theorems are obtained. Furthermore, we also consider the iterative algorithms with perturbations for finding a common element of the solution set of the system of generalized equilibrium problems and the common fixed point set of the super hybrid mappings in a Hilbert space.

\section{Preliminaries}

Let $H$ be a real Hilbert space with inner product $\langle\cdot, \cdot\rangle$ and norm $\|\cdot\|$. We denote the strongly convergence and the weak convergence of $\left\{x_{n}\right\}$ to $x \in H$ by $x_{n} \rightarrow x$ and $x_{n} \rightarrow x$, respectively. In a Hilbert space, it is known that

$$
\|\lambda x+(1-\lambda) y\|^{2}=\lambda\|x\|^{2}+(1-\lambda)\|y\|^{2}-\lambda(1-\lambda)\|x-y\|^{2},
$$

for all $x, y \in H$ and $\lambda \in \mathbb{R}$; see [19]. Furthermore, we have that for any $x, y, u, v \in H$

$$
2\langle x-y, u-v\rangle=\|x-v\|^{2}+\|y-u\|^{2}-\|x-u\|^{2}-\|y-v\|^{2} .
$$

Let $C$ be a nonempty closed convex subset of $H$ and $x \in H$. We know that there exists a unique nearest point $z \in C$ such that $\|x-z\|=\inf _{y \in C}\|x-y\|$. We denote such a correspondence by $z=P_{C} x$. The mapping $P_{C}$ is called the metric projection of $H$ onto $C$. It is known that $P_{C}$ is nonexpansive and

$$
\left\langle x-P_{C} x, P_{C} x-u\right\rangle \geq 0
$$

for all $x \in H$ and $u \in C$; see $[19,20]$ for more details.

Let $C$ be a nonempty, closed and convex subset of $H$ and let $G: C \times C \rightarrow \mathbb{R}$ be a bifunction. For solving the generalized equilibrium problem, let us assume that the bifunction $G: C \times C \rightarrow \mathbb{R}$ satisfies the following conditions:

(A1) $G(x, x)=0$ for a $11 x \in C$;

(A2) $G$ is monotone, that is, $G(x, y)+G(y, x) \leq 0$ for any $x, y \in C$;

(A3) for each $x, y, z \in C$

$$
\lim _{t \downarrow 0} G(t z+(1-t) x, y) \leq G(x, y)
$$

(A4) for each $x \in C, G(x, \cdot)$ is convex and lower semicontinuous.

We know the following lemma which appears implicitly in Blum and Oettli [4].

Lemma 2.1 (see [4]). Let $C$ be a nonempty closed convex subset of $H$ and let $G$ be a bifunction of $C \times C$ into $\mathbb{R}$ satisfying (A1)-(A4). Let $r>0$ and $x \in H$. Then, there exists a unique $z \in C$ such that

$$
G(z, y)+\frac{1}{r}\langle y-z, z-x\rangle \geq 0, \quad \forall y \in C .
$$

The following lemma was also given in Combettes and Hirstoaga [5]. 
Lemma 2.2 (see [5]). Let $C$ be a nonempty closed convex subset of a real Hilbert space $H$. Let $G: C \times C \rightarrow \mathbb{R}$ be a bifunction which satisfies conditions (A1)-(A4). For $r>0$ and $x \in H$, define a mapping $T_{r}: H \rightarrow C$ as follows:

$$
T_{r}(x)=\left\{z \in C: G(z, y)+\frac{1}{r}\langle y-z, z-x\rangle \geq 0, \quad \forall y \in C\right\}
$$

for all $x \in H$. Then the following hold:

(i) $T_{r}$ is single-valued;

(ii) $T_{r}$ is firmly nonexpansive, that is, for any $x, y \in C$,

$$
\left\|T_{r} x-T_{r} y\right\|^{2} \leq\left\langle T_{r} x-T_{r} y, x-y\right\rangle
$$

(iii) $(E P)$ is a closed convex subset of $C$;

(iv) $F\left(T_{r}\right)=E P(G)$.

Remark 2.3. For any $x \in H$ and $r>0$, by Lemma 2.2 (i), there exists $u \in H$ such that

$$
G(u, y)+\frac{1}{r}\langle y-u, u-x\rangle \geq 0, \quad \forall y \in H
$$

Replacing $x$ with $x-r \Psi x \in H$ in (2.8), we have

$$
G(u, y)+\langle\Psi x, y-u\rangle+\frac{1}{r}\langle y-u, u-x\rangle \geq 0, \quad \forall y \in H
$$

where $\Psi: H \rightarrow H$ is an inverse strongly monotone mapping.

Lemma 2.4 (see [21]). Let $\left\{\Gamma_{n}\right\}$ be a sequence of real numbers that does not decrease at infinity in the sense that there exists a subsequence $\left\{\Gamma_{n_{i}}\right\}$ of $\left\{\Gamma_{n}\right\}$ which satisfies $\Gamma_{n_{i}}<\Gamma_{n_{i}+1}$ for all $i \in \mathbb{N}$. Define the sequence $\{\tau(n)\}_{n \geq n_{0}}$ of integers as follows:

$$
\tau(n)=\max \left\{k \leq n: \Gamma_{k}<\Gamma_{k+1}\right\},
$$

where $n_{0} \in \mathbb{N}$ such that $\left\{k \leq n_{0}: \Gamma_{k}<\Gamma_{k+1}\right\} \neq \emptyset$. Then, the following hold:

(i) $\tau(1) \leq \tau(2) \leq \cdots$ and $\tau(n) \rightarrow \infty$;

(ii) $\Gamma_{\tau(n)} \leq \Gamma_{\tau(n)+1}$ and $\Gamma_{n} \leq \Gamma_{\tau(n)+1}, \forall n \in \mathbb{N}$.

Lemma 2.5 (see [22]). Let $\left\{a_{n}\right\}_{n \in \mathbb{N}}$ be a sequence of nonnegative real numbers, $\left\{\alpha_{n}\right\}$ a sequence of real numbers in $[0,1]$ with $\sum_{n=1}^{\infty} \alpha_{n}=\infty,\left\{u_{n}\right\}$ a sequence of nonnegative real numbers with $\sum_{n=1}^{\infty} u_{n}<\infty,\left\{t_{n}\right\}$ a sequence of real numbers with lim sup $t_{n} \leq 0$. Suppose that

$$
a_{n+1} \leq\left(1-\alpha_{n}\right) a_{n}+\alpha_{n} t_{n}+u_{n}, \quad \forall n \in \mathbb{N} .
$$

Then $\lim _{n \rightarrow \infty} a_{n}=0$. 


\section{Main Results}

Let $C$ be a nonempty closed convex subset of a Hilbert space $H$. For each $i=1,2, \ldots, k$, let $G_{i}: C \times C \rightarrow \mathbb{R}$ be a bifunction satisfying (A1)-(A4) and $\Psi_{i}$ a $\mu_{i}$-inverse strongly monotone mapping. For each $j=1,2$, let $T_{j}: C \rightarrow H$ be two mappings. Let $\left\{x_{n}\right\}$ be a sequence generated in the following manner:

$$
\begin{gathered}
x_{1} \in H, \\
G_{1}\left(u_{n, 1}, y\right)+\left\langle\Psi_{1} x_{n}, y-u_{n, 1}\right\rangle+\frac{1}{r_{n}}\left\langle y-u_{n, 1}, u_{n, 1}-x_{n}\right\rangle \geq 0, \quad \forall y \in C, \\
G_{2}\left(u_{n, 2}, y\right)+\left\langle\Psi_{2} x_{n}, y-u_{n, 2}\right\rangle+\frac{1}{r_{n}}\left\langle y-u_{n, 2}, u_{n, 2}-x_{n}\right\rangle \geq 0, \quad \forall y \in C, \\
\vdots \\
G_{k}\left(u_{n, k}, y\right)+\left\langle\Psi_{k} x_{n}, y-u_{n, k}\right\rangle+\frac{1}{r_{n}}\left\langle y-u_{n, k}, u_{n, k}-x_{n}\right\rangle \geq 0, \quad \forall y \in C, \\
\omega_{n}=\frac{1}{k} \sum_{i=1}^{k} u_{n, i}, \\
y_{n}=\gamma_{n} \omega_{n}+\left(1-\gamma_{n}\right) T_{1} \omega_{n}, \\
z_{n}=\beta_{n} y_{n}+\left(1-\beta_{n}\right) T_{2} \omega_{n}, \\
x_{n+1}=\alpha_{n} u_{n}+\left(1-\alpha_{n}\right) z_{n}, \quad \forall n \in \mathbb{N},
\end{gathered}
$$

where $\left\{\alpha_{n}\right\},\left\{\beta_{n}\right\},\left\{\gamma_{n}\right\}$ are sequences in $(0,1)$ and $\left\{u_{n}\right\} \subset H$ is a sequence and $\left\{r_{n}\right\} \subset\left[a, 2 \mu_{i}\right)$ for some $a>0$ and for all $i \in\{1,2, \ldots, k\}$. Under certain appropriate assumptions imposed on the sequences $\left\{\alpha_{n}\right\},\left\{\beta_{n}\right\},\left\{\gamma_{n}\right\}$, the strong convergence theorem of $\left\{x_{n}\right\}$ defined by (3.1) is studied in the following theorem.

Theorem 3.1. Let $C$ be a nonempty closed convex subset of a Hilbert space $H$. For each $i=1,2, \ldots, k$, let $G_{i}: C \times C \rightarrow \mathbb{R}$ be a bifunction satisfying (A1)-(A4) and $\Psi_{i}$ a $\mu_{i}$-inverse strongly monotone mapping. For each $j=1,2$, let $T_{j}: C \rightarrow H$ be two quasi-nonexpansive mappings such that $I-T_{j}$ are demiclosed at zero with $\Omega:=F\left(T_{1}\right) \cap F\left(T_{2}\right) \cap\left(\cap_{i=1}^{k} \operatorname{GEP}\left(G_{i}, \Psi_{i}\right)\right) \neq \emptyset$. Let the sequences $\left\{x_{n}\right\},\left\{y_{n}\right\}$, and $\left\{z_{n}\right\}$ be defined by (3.1), where $\left\{\alpha_{n}\right\},\left\{\beta_{n}\right\},\left\{\gamma_{n}\right\}$, and $\left\{u_{n}\right\}$ satisfy the following conditions:

(C1) $\lim _{n \rightarrow \infty} \alpha_{n}=0$ and $\sum_{n=1}^{\infty} \alpha_{n}=\infty$;

(C2) $\liminf _{n \rightarrow \infty} \beta_{n}\left(1-\beta_{n}\right)>0$;

(C3) $\liminf _{n \rightarrow \infty} \gamma_{n}\left(1-\gamma_{n}\right)>0$;

(C4) $\lim _{n \rightarrow \infty} u_{n}=u$ for some $u \in H$.

Then $\left\{x_{n}\right\}$ converges strongly to $x^{*}$, where $x^{*}=P_{\Omega} u$. 
Proof. We first have that for all $i=1,2, \ldots, k, I-r_{n} \Psi_{i}$ is a nonexpansive mapping. Indeed, for all $x, y \in C$, we obtain

$$
\begin{aligned}
\left\|\left(I-r_{n} \Psi_{i}\right) x-\left(I-r_{n} \Psi_{i}\right) y\right\|^{2} & =\left\|(x-y)-r_{n}\left(\Psi_{i} x-\Psi_{i} y\right)\right\|^{2} \\
& =\|x-y\|^{2}-2 r_{n}\left\langle\Psi_{i} x-\Psi_{i} y, x-y\right\rangle+r_{n}^{2}\left\|\Psi_{i} x-\Psi_{i} y\right\|^{2} \\
& \leq\|x-y\|^{2}-r_{n}\left(2 \mu_{i}-r_{n}\right)\left\|\Psi_{i} x-\Psi_{i} y\right\|^{2} \\
& \leq\|x-y\|^{2} .
\end{aligned}
$$

Thus $I-r_{n} \Psi_{i}$ is nonexpansive for each $i \in\{1,2, \ldots, k\}$. Now, let $w \in \Omega$ be arbitrary. By (C4), $\left\{u_{n}\right\}$ is a bounded sequence, there exists $M \leq 0$ such that

$$
\sup _{n \in \mathbb{N}}\left\|u_{n}-w\right\| \leq M
$$

For each $i=1,2, \ldots, k$ and $n \in \mathbb{N}$, we have from $u_{n, i}=T_{r_{n, i}}\left(x_{n}-r_{n} \Psi_{i} x_{n}\right)$ that

$$
\begin{aligned}
\left\|u_{n, i}-w\right\| & =\left\|T_{r_{n, i}}\left(x_{n}-r_{n} \Psi_{i} x_{n}\right)-T_{r_{n, i}}\left(w-r_{n} \Psi_{i} w\right)\right\| \\
& \leq\left\|\left(x_{n}-r_{n} \Psi_{i} x_{n}\right)-\left(w-r_{n} \Psi_{i} w\right)\right\| \\
& \leq\left\|x_{n}-w\right\|,
\end{aligned}
$$

which gives also that

$$
\left\|\omega_{n}-w\right\| \leq \frac{1}{k} \sum_{i=1}^{k}\left\|u_{n, i}-w\right\| \leq\left\|x_{n}-w\right\| \quad \forall w \in \Omega .
$$

Since $T_{1}$ is quasi-nonexpansive, we have

$$
\begin{aligned}
\left\|y_{n}-w\right\| & =\left\|\gamma_{n} \omega_{n}+\left(1-\gamma_{n}\right) T_{1} \omega_{n}-w\right\| \\
& =\left\|\gamma_{n}\left(\omega_{n}-w\right)+\left(1-\gamma_{n}\right)\left(T_{1} \omega_{n}-w\right)\right\| \\
& \leq \gamma_{n}\left\|\omega_{n}-w\right\|+\left(1-\gamma_{n}\right)\left\|T_{1} \omega_{n}-w\right\| \\
& \leq\left\|\omega_{n}-w\right\| .
\end{aligned}
$$

So, we have from (3.5) and (3.6) and the quasi-nonexpansiveness of $T_{2}$ that

$$
\begin{aligned}
\left\|x_{n+1}-w\right\| & =\left\|\alpha_{n}\left(u_{n}-w\right)+\left(1-\alpha_{n}\right)\left(z_{n}-w\right)\right\| \\
& \leq \alpha_{n}\left\|u_{n}-w\right\|+\left(1-\alpha_{n}\right)\left\|z_{n}-w\right\| \\
& \leq \alpha_{n}\left\|u_{n}-w\right\|+\left(1-\alpha_{n}\right)\left\{\beta_{n}\left\|y_{n}-w\right\|+\left(1-\beta_{n}\right)\left\|T_{2} \omega_{n}-w\right\|\right\} \\
& \leq \alpha_{n}\left\|u_{n}-w\right\|+\left(1-\alpha_{n}\right)\left\{\beta_{n}\left\|\omega_{n}-w\right\|+\left(1-\beta_{n}\right)\left\|\omega_{n}-w\right\|\right\} \\
& \leq \alpha_{n}\left\|u_{n}-w\right\|+\left(1-\alpha_{n}\right)\left\|\omega_{n}-w\right\| \\
& \leq \alpha_{n}\left\|u_{n}-w\right\|+\left(1-\alpha_{n}\right)\left\|x_{n}-w\right\| \\
& \leq \max \left\{M,\left\|x_{n}-w\right\|\right\} .
\end{aligned}
$$


By Induction, we have that

$$
\left\|x_{n}-w\right\| \leq \max \left\{\left\|x_{1}-w\right\|, M\right\}, \quad \forall n \in \mathbb{N} .
$$

Thus we obtain that $\left\{\left\|x_{n}-w\right\|\right\}$ is bounded, so also $\left\{x_{n}\right\},\left\{y_{n}\right\},\left\{z_{n}\right\},\left\{\omega_{n}\right\},\left\{T_{1} \omega_{n}\right\}$, and $\left\{T_{2} \omega_{n}\right\}$ are bounded. Since $\Omega$ is closed and convex, we can take $x^{*}=P_{\Omega} u$. It follows that

$$
\begin{aligned}
\left\|y_{n}-x^{*}\right\|^{2} & =\left\|\gamma_{n}\left(\omega_{n}-x^{*}\right)+\left(1-\gamma_{n}\right)\left(T_{1} \omega_{n}-x^{*}\right)\right\|^{2} \\
& =\gamma_{n}\left\|\omega_{n}-x^{*}\right\|^{2}+\left(1-\gamma_{n}\right)\left\|T_{1} \omega_{n}-x^{*}\right\|^{2}-\gamma_{n}\left(1-\gamma_{n}\right)\left\|\omega_{n}-T_{1} \omega_{n}\right\|^{2} \\
& \leq \gamma_{n}\left\|\omega_{n}-x^{*}\right\|^{2}+\left(1-\gamma_{n}\right)\left\|\omega_{n}-x^{*}\right\|^{2}-\gamma_{n}\left(1-\gamma_{n}\right)\left\|\omega_{n}-T_{1} \omega_{n}\right\|^{2} \\
& =\left\|\omega_{n}-x^{*}\right\|^{2}-\gamma_{n}\left(1-\gamma_{n}\right)\left\|\omega_{n}-T_{1} \omega_{n}\right\|^{2} \\
& \leq\left\|\omega_{n}-x^{*}\right\|^{2} .
\end{aligned}
$$

From (3.9), we have

$$
\begin{aligned}
\left\|z_{n}-x^{*}\right\|^{2} & =\left\|\beta_{n}\left(y_{n}-x^{*}\right)+\left(1-\beta_{n}\right)\left(T_{2} \omega_{n}-x^{*}\right)\right\|^{2} \\
& =\beta_{n}\left\|y_{n}-x^{*}\right\|^{2}+\left(1-\beta_{n}\right)\left\|T_{2} \omega_{n}-x^{*}\right\|^{2}-\beta_{n}\left(1-\beta_{n}\right)\left\|y_{n}-T_{2} \omega_{n}\right\|^{2} \\
& \leq \beta_{n}\left\|\omega_{n}-x^{*}\right\|^{2}+\left(1-\beta_{n}\right)\left\|\omega_{n}-x^{*}\right\|^{2}-\beta_{n}\left(1-\beta_{n}\right)\left\|y_{n}-T_{2} \omega_{n}\right\|^{2} \\
& =\left\|\omega_{n}-x^{*}\right\|^{2}-\beta_{n}\left(1-\beta_{n}\right)\left\|y_{n}-T_{2} \omega_{n}\right\|^{2} \\
& \leq\left\|\omega_{n}-x^{*}\right\|^{2} .
\end{aligned}
$$

Hence we have from (3.5), (3.9), and (3.10) that

$$
\begin{aligned}
\left\|\omega_{n+1}-x^{*}\right\|^{2} & \leq\left\|x_{n+1}-x^{*}\right\|^{2} \\
& =\left\|\alpha_{n}\left(u_{n}-x^{*}\right)+\left(1-\alpha_{n}\right)\left(z_{n}-x^{*}\right)\right\|^{2} \\
& =\alpha_{n}\left\|u_{n}-x^{*}\right\|^{2}+\left(1-\alpha_{n}\right)\left\|z_{n}-x^{*}\right\|^{2}-\alpha_{n}\left(1-\alpha_{n}\right)\left\|u_{n}-z_{n}\right\|^{2} \\
& \leq \alpha_{n}\left\|u_{n}-x^{*}\right\|^{2}+\left(1-\alpha_{n}\right)\left\|z_{n}-x^{*}\right\|^{2} \\
& =\alpha_{n}\left\|u_{n}-x^{*}\right\|^{2}+\left(1-\alpha_{n}\right)\left\{\beta_{n}\left\|y_{n}-x^{*}\right\|^{2}+\left(1-\beta_{n}\right)\left\|T_{2} \omega_{n}-x^{*}\right\|^{2}\right. \\
& \left.\quad-\beta_{n}\left(1-\beta_{n}\right)\left\|y_{n}-T_{2} \omega_{n}\right\|^{2}\right\} \\
& \leq \alpha_{n}\left\|u_{n}-x^{*}\right\|^{2}+\beta_{n}\left\{\gamma_{n}\left\|\omega_{n}-x^{*}\right\|^{2}+\left(1-\gamma_{n}\right)\left\|T_{1} \omega_{n}-x^{*}\right\|^{2}\right. \\
& \left.\quad-\gamma_{n}\left(1-\gamma_{n}\right)\left\|\omega_{n}-T_{1} \omega_{n}\right\|^{2}\right\}+\left(1-\beta_{n}\right)\left\|T_{2} \omega_{n}-x^{*}\right\|^{2} \\
& -\beta_{n}\left(1-\beta_{n}\right)\left\|y_{n}-T_{2} \omega_{n}\right\|^{2}
\end{aligned}
$$


Abstract and Applied Analysis

$$
\begin{aligned}
\leq & \alpha_{n}\left\|u_{n}-x^{*}\right\|^{2}+\beta_{n}\left(\gamma_{n}\left\|\omega_{n}-x^{*}\right\|^{2}+\left(1-\gamma_{n}\right)\left\|\omega_{n}-x^{*}\right\|^{2}\right. \\
& \left.\quad-\gamma_{n}\left(1-\gamma_{n}\right)\left\|\omega_{n}-T_{1} \omega_{n}\right\|^{2}\right)+\left(1-\beta_{n}\right)\left\|\omega_{n}-x^{*}\right\|^{2} \\
& -\beta_{n}\left(1-\beta_{n}\right)\left\|y_{n}-T_{2} \omega_{n}\right\|^{2} \\
= & \alpha_{n}\left\|u_{n}-x^{*}\right\|^{2}+\left\|\omega_{n}-x^{*}\right\|^{2}-\gamma_{n}\left(1-\gamma_{n}\right)\left\|\omega_{n}-T_{1} \omega_{n}\right\|^{2} \\
& -\beta_{n}\left(1-\beta_{n}\right)\left\|y_{n}-T_{2} \omega_{n}\right\|^{2} .
\end{aligned}
$$

We also have that

$$
\begin{aligned}
& \gamma_{n}\left(1-\gamma_{n}\right)\left\|\omega_{n}-T_{1} \omega_{n}\right\|^{2} \leq \alpha_{n}\left\|u_{n}-x^{*}\right\|^{2}+\left\|\omega_{n}-x^{*}\right\|^{2}-\left\|\omega_{n+1}-x^{*}\right\|^{2}, \\
& \beta_{n}\left(1-\beta_{n}\right)\left\|y_{n}-T_{2} \omega_{n}\right\|^{2} \leq \alpha_{n}\left\|u_{n}-x^{*}\right\|^{2}+\left\|\omega_{n}-x^{*}\right\|^{2}-\left\|\omega_{n+1}-x^{*}\right\|^{2} .
\end{aligned}
$$

Furthemore, we have from $y_{n}=\gamma_{n} \omega_{n}+\left(1-\gamma_{n}\right) T_{1} \omega_{n}$ that

$$
\begin{aligned}
\left\|\omega_{n}-T_{2} \omega_{n}\right\| & \leq\left\|\omega_{n}-y_{n}\right\|+\left\|y_{n}-T_{2} \omega_{n}\right\| \\
& =\left\|\omega_{n}-\gamma_{n} \omega_{n}-\left(1-\gamma_{n}\right) T_{1} \omega_{n}\right\|+\left\|y_{n}-T_{2} x_{n}\right\| \\
& =\left(1-\gamma_{n}\right)\left\|\omega_{n}-T_{1} \omega_{n}\right\|+\left\|y_{n}-T_{2} \omega_{n}\right\| .
\end{aligned}
$$

On the other hand, since $x_{n+1}-x^{*}=\alpha_{n}\left(u_{n}-x^{*}\right)+\left(1-\alpha_{n}\right)\left(z_{n}-x^{*}\right)$, we have

$$
\begin{aligned}
\left\|\omega_{n+1}-x^{*}\right\|^{2} \leq & \left\|x_{n+1}-x^{*}\right\|^{2} \\
\leq & \left(1-\alpha_{n}\right)\left\|z_{n}-x^{*}\right\|^{2}+2 \alpha_{n}\left\langle u_{n}-x^{*}, x_{n+1}-x^{*}\right\rangle \\
\leq & \left(1-\alpha_{n}\right)\left\|\omega_{n}-x^{*}\right\|^{2}+2 \alpha_{n}\left\langle u_{n}-x^{*}, x_{n+1}-x^{*}\right\rangle \\
= & \left(1-\alpha_{n}\right)\left\|\omega_{n}-x^{*}\right\|^{2}+2 \alpha_{n}\left\langle u_{n}-u, x_{n+1}-x^{*}\right\rangle \\
& +2 \alpha_{n}\left\langle u-x^{*}, x_{n+1}-x^{*}\right\rangle \\
= & \left(1-\alpha_{n}\right)\left\|\omega_{n}-x^{*}\right\|^{2}+2 \alpha_{n}\left\langle u_{n}-u, x_{n+1}-x^{*}\right\rangle \\
& +2 \alpha_{n}\left\langle u-x^{*}, x_{n+1}-\omega_{n}\right\rangle+2 \alpha_{n}\left\langle u-x^{*}, \omega_{n}-x^{*}\right\rangle .
\end{aligned}
$$

We also have that

$$
\begin{aligned}
\left\|x_{n+1}-\omega_{n}\right\| \leq & \left\|x_{n+1}-y_{n}\right\|+\left\|y_{n}-\omega_{n}\right\| \\
= & \left\|\alpha_{n}\left(u_{n}-y_{n}\right)+\left(1-\alpha_{n}\right)\left(z_{n}-y_{n}\right)\right\|+\left\|\left(1-\gamma_{n}\right)\left(\omega_{n}-T_{1} \omega_{n}\right)\right\| \\
\leq & \alpha_{n}\left\|u_{n}-y_{n}\right\|+\left(1-\alpha_{n}\right)\left\|\beta_{n} y_{n}+\left(1-\beta_{n}\right) T_{2} \omega_{n}-y_{n}\right\| \\
& +\left(1-\gamma_{n}\right)\left\|\omega_{n}-T_{1} \omega_{n}\right\| \\
= & \alpha_{n}\left\|u_{n}-y_{n}\right\|+\left(1-\alpha_{n}\right)\left(1-\beta_{n}\right)\left\|y_{n}-T_{2} \omega_{n}\right\| \\
& +\left(1-\gamma_{n}\right)\left\|\omega_{n}-T_{1} \omega_{n}\right\| .
\end{aligned}
$$


Moreover, for any $i \in\{1,2, \ldots, k\}$, we have from $u_{n, i}=T_{r_{n, i}}\left(x_{n}-r_{n} \Psi_{i} x_{n}\right)$ that

$$
\begin{aligned}
\left\|u_{n, i}-x^{*}\right\|^{2} & \leq\left\|\left(x_{n}-x^{*}\right)-r_{n}\left(\Psi_{i} x_{n}-\Psi_{i} x^{*}\right)\right\|^{2} \\
& =\left\|x_{n}-x^{*}\right\|^{2}-2 r_{n}\left\langle x_{n}-x^{*}, \Psi_{i} x_{n}-\Psi_{i} x^{*}\right\rangle+r_{n}^{2}\left\|\Psi_{i} x_{n}-\Psi_{i} x^{*}\right\|^{2} \\
& \leq\left\|x_{n}-x^{*}\right\|^{2}-r_{n}\left(2 \mu_{i}-r_{n}\right)\left\|\Psi_{i} x_{n}-\Psi_{i} x^{*}\right\|^{2} .
\end{aligned}
$$

It follows that

$$
\begin{aligned}
\left\|\omega_{n}-x^{*}\right\|^{2} & =\left\|\sum_{i=1}^{k} \frac{1}{k}\left(u_{n, i}-x^{*}\right)\right\|^{2} \\
& \leq \frac{1}{k} \sum_{i=1}^{k}\left\|u_{n, i}-x^{*}\right\|^{2} \\
& \leq\left\|x_{n}-x^{*}\right\|^{2}-\frac{1}{k} \sum_{i=1}^{k} r_{n}\left(2 \mu_{i}-r_{n}\right)\left\|\Psi_{i} x_{n}-\Psi_{i} x^{*}\right\|^{2} .
\end{aligned}
$$

This implies that

$$
\begin{aligned}
\left\|x_{n+1}-x^{*}\right\|^{2}= & \left\|\alpha_{n}\left(u_{n}-x^{*}\right)+\left(1-\alpha_{n}\right)\left(z_{n}-x^{*}\right)\right\|^{2} \\
\leq & \alpha_{n}\left\|u_{n}-x^{*}\right\|^{2}+\left(1-\alpha_{n}\right)\left\|\omega_{n}-x^{*}\right\|^{2} \\
\leq & \alpha_{n}\left\|u_{n}-x^{*}\right\|^{2}+\left(1-\alpha_{n}\right)\left\|x_{n}-x^{*}\right\|^{2} \\
& -\left(1-\alpha_{n}\right) \frac{1}{k} \sum_{i=1}^{k} r_{n}\left(2 \mu_{i}-r_{n}\right)\left\|\Psi_{i} x_{n}-\Psi_{i} x^{*}\right\|^{2},
\end{aligned}
$$

and hence

$$
\left(1-\alpha_{n}\right) \frac{1}{k} \sum_{i=1}^{k} r_{n}\left(2 \mu_{i}-r_{n}\right)\left\|\Psi_{i} x_{n}-\Psi_{i} x^{*}\right\|^{2} \leq \alpha_{n}\left\|u_{n}-x^{*}\right\|^{2}+\left\|x_{n}-x^{*}\right\|^{2}-\left\|x_{n+1}-x^{*}\right\|^{2}
$$

Furthermore, we have from Lemma 2.2 that for any $i \in 1,2, \ldots, k$, we have

$$
\begin{aligned}
\left\|u_{n, i}-x^{*}\right\|^{2} \leq & \left\langle\left(x_{n}-r_{n} \Psi_{i} x_{n}\right)-\left(x^{*}-r_{n} \Psi_{i} x^{*}\right), u_{n, i}-x^{*}\right\rangle \\
= & \frac{1}{2}\left\{\left\|\left(x_{n}-r_{n} \Psi_{i} x_{n}\right)-\left(x^{*}-r_{n} \Psi_{i} x^{*}\right)\right\|^{2}+\left\|u_{n, i}-x^{*}\right\|^{2}\right. \\
& \left.\quad-\left\|\left(x_{n}-r_{n} \Psi_{i} x_{n}\right)-\left(x^{*}-r_{n} \Psi_{i} x^{*}\right)-\left(u_{n, i}-x^{*}\right)\right\|^{2}\right\} \\
\leq & \frac{1}{2}\left\{\left\|x_{n}-x^{*}\right\|^{2}+\left\|u_{n, i}-x^{*}\right\|^{2}-\left\|\left(x_{n}-u_{n, i}\right)-r_{n}\left(\Psi_{i} x_{n}-\Psi_{i} x^{*}\right)\right\|^{2}\right\} \\
= & \frac{1}{2}\left\{\left\|x_{n}-x^{*}\right\|^{2}+\left\|u_{n, i}-x^{*}\right\|^{2}-\left\|x_{n}-u_{n, i}\right\|^{2}-r_{n}^{2}\left\|\Psi_{i} x_{n}-\Psi_{i} x^{*}\right\|^{2}\right. \\
& \left.\quad+2 r_{n}\left\langle x_{n}-u_{n, i}, \Psi_{i} x_{n}-\Psi_{i} x^{*}\right\rangle\right\} .
\end{aligned}
$$


This implies that

$$
\left\|u_{n, i}-x^{*}\right\|^{2} \leq\left\|x_{n}-x^{*}\right\|^{2}-\left\|x_{n}-u_{n, i}\right\|^{2}+2 r_{n}\left\|x_{n}-u_{n, i}\right\|\left\|\Psi_{i} x_{n}-\Psi_{i} x^{*}\right\|
$$

Then we have from (3.22) that

$$
\begin{aligned}
\left\|\omega_{n}-x^{*}\right\|^{2} & \leq \frac{1}{k} \sum_{i=1}^{k}\left\|u_{n, i}-x^{*}\right\|^{2} \\
& \leq\left\|x_{n}-x^{*}\right\|^{2}-\frac{1}{k} \sum_{i=1}^{k}\left\|u_{n, i}-x_{n}\right\|^{2} \\
& +\frac{1}{k} \sum_{i=1}^{k} 2 r_{n}\left\|x_{n}-u_{n, i}\right\|\left\|\Psi_{i} x_{n}-\Psi_{i} x^{*}\right\| .
\end{aligned}
$$

Hence we have from (3.23) that

$$
\begin{aligned}
\left\|x_{n+1}-x^{*}\right\|^{2} \leq & \alpha_{n}\left\|u_{n}-x^{*}\right\|^{2}+\left(1-\alpha_{n}\right)\left\|\omega_{n}-x^{*}\right\|^{2} \\
\leq & \alpha_{n}\left\|u_{n}-x^{*}\right\|^{2}+\left(1-\alpha_{n}\right)\left(\left\|x_{n}-x^{*}\right\|^{2}-\frac{1}{k} \sum_{i=1}^{k}\left\|u_{n, i}-x_{n}\right\|^{2}\right) \\
& +\left(1-\alpha_{n}\right)\left(\frac{1}{k} \sum_{i=1}^{k} 2 r_{n}\left\|x_{n}-u_{n, i}\right\|\left\|\Psi_{i} x_{n}-\Psi_{i} x^{*}\right\|\right) .
\end{aligned}
$$

It follows that

$$
\begin{aligned}
\left(1-\alpha_{n}\right) \frac{1}{k} \sum_{i=1}^{k}\left\|u_{n, i}-x_{n}\right\|^{2} \leq & \alpha_{n}\left\|u_{n}-x^{*}\right\|^{2}+\left\|x_{n}-x^{*}\right\|^{2}-\left\|x_{n+1}-x^{*}\right\|^{2} \\
& +\left(1-\alpha_{n}\right)\left(\frac{1}{k} \sum_{i=1}^{k} 2 r_{n}\left\|x_{n}-u_{n, i}\right\|\left\|\Psi_{i} x_{n}-\Psi_{i} x^{*}\right\|\right) .
\end{aligned}
$$

Next, we will consider the following two cases.

Case A. Put $\Gamma_{n}=\left\|\omega_{n}-x^{*}\right\|^{2}$ for all $n \in \mathbb{N}$. Suppose that $\Gamma_{n+1} \leq \Gamma_{n}$ for all $n \in \mathbb{N}$. In this case $\lim _{n \rightarrow \infty} \Gamma_{n}$ exists and then $\lim _{n \rightarrow \infty}\left(\Gamma_{n+1}-\Gamma_{n}\right)=0$. By (C1), (C3), and (3.12), we have

$$
\lim _{n \rightarrow \infty}\left\|\omega_{n}-T_{1} \omega_{n}\right\|=0 .
$$

Similarly by (C1), (C2), and (3.13), we also have

$$
\lim _{n \rightarrow \infty}\left\|y_{n}-T_{2} \omega_{n}\right\|=0 .
$$


So, we have from (3.14), (3.26), and (3.27) that

$$
\lim _{n \rightarrow \infty}\left\|\omega_{n}-T_{2} \omega_{n}\right\|=0 .
$$

Since $\lim _{n \rightarrow \infty}\left\|\omega_{n}-x^{*}\right\|$ exists, we have from (3.11) and (3.26)

$$
\lim _{n \rightarrow \infty}\left\|\omega_{n}-x^{*}\right\|=\lim _{n \rightarrow \infty}\left\|x_{n}-x^{*}\right\| .
$$

We also have from (C1), (3.16), (3.26), and (3.27) that

$$
\lim _{n \rightarrow \infty}\left\|x_{n+1}-\omega_{n}\right\|=0 .
$$

Since $\lim _{n \rightarrow \infty}\left\|x_{n}-x^{*}\right\|$ exists we have from (C1) and (3.20) that

$$
\lim _{n \rightarrow \infty}\left\|\Psi_{i} x_{n}-\Psi_{i} x^{*}\right\|=0, \quad \forall i=1,2, \ldots, k .
$$

This together with (3.25) and the existence of $\lim _{n \rightarrow \infty}\left\|x_{n}-x^{*}\right\|$ implies that

$$
\lim _{n \rightarrow \infty}\left\|u_{n, i}-x_{n}\right\|=0, \quad \forall i=1,2, \ldots, k,
$$

which gives that

$$
\left\|\omega_{n}-x_{n}\right\| \leq \frac{1}{k} \sum_{i=1}^{k}\left\|u_{n, i}-x_{n}\right\| \longrightarrow 0 \quad \text { as } n \longrightarrow \infty
$$

So, from (3.30), $\lim _{n \rightarrow \infty}\left\|x_{n+1}-x_{n}\right\|=0$. Furthermore, we have from (3.33) that

$$
\left\|\omega_{n+1}-\omega_{n}\right\| \leq\left\|\omega_{n+1}-x_{n+1}\right\|+\left\|x_{n+1}-x_{n}\right\|+\left\|x_{n}-\omega_{n}\right\| \longrightarrow 0 \quad \text { as } n \longrightarrow \infty ;
$$

that is

$$
\lim _{n \rightarrow \infty}\left\|\omega_{n+1}-\omega_{n}\right\|=0 .
$$

Now, since $\left\{\omega_{n}\right\}$ is a bounded sequence, there exists a subsequence $\left\{\omega_{n_{j}}\right\}$ of $\left\{\omega_{n}\right\}$ such that

$$
\limsup _{n \rightarrow \infty}\left\langle u-x^{*}, \omega_{n}-x^{*}\right\rangle=\lim _{j \rightarrow \infty}\left\langle u-x^{*}, \omega_{n_{j}}-x^{*}\right\rangle .
$$

Without loss of generality, we may assume that $\omega_{n_{j}} \rightarrow v$. Since $T_{1}$ is demiclosed at zero and by (3.26), we conclude that $v \in F\left(T_{1}\right)$. Similarly, since $T_{2}$ is demiclosed at zero and by (3.28), we have $v \in F\left(T_{2}\right)$. Therefore, we get that

$$
v \in F\left(T_{1}\right) \cap F\left(T_{2}\right) .
$$


Next, we show that $v \in \cap_{i=1}^{k} \operatorname{GEP}\left(G_{i}, \Psi_{i}\right)$. For each $i \in\{1,2, \ldots, k\}$, since $u_{n, i}=T_{r_{n, i}}\left(x_{n}-\right.$ $\left.r_{n} \Psi_{i} x_{n}\right)$, we have

$$
G_{i}\left(u_{n, i}, y\right)+\left\langle\Psi_{i} x_{n}, y-u_{n, i}\right\rangle+\frac{1}{r_{n}}\left\langle y-u_{n, i}, u_{n, i}-x_{n}\right\rangle \geq 0, \quad \forall y \in C
$$

From (A2), we also have

$$
\left\langle\Psi_{i} x_{n}, y-u_{n, i}\right\rangle+\frac{1}{r_{n}}\left\langle y-u_{n, i}, u_{n, i}-x_{n}\right\rangle \geq G_{i}\left(y, u_{n, i}\right) .
$$

Replacing $n$ by $n_{j}$, we have

$$
\left\langle\Psi_{i} x_{n_{j}}, y-u_{n_{j}, i}\right\rangle+\left\langle y-u_{n_{j}, i}, \frac{u_{n_{j}, i}-x_{n_{j}}}{r_{n_{j}}}\right\rangle \geq G_{i}\left(y, u_{n_{j}, i}\right) .
$$

Put $y_{t}=t y+(1-t) v$ for all $t \in(0,1]$ and $y \in C$. Since $v \in C$, then $y_{t} \in C$ and

$$
\begin{aligned}
\left\langle y_{t}-u_{n_{j}, i}, \Psi_{i} y_{t}\right\rangle \geq & \left\langle y_{t}-u_{n_{j}, i}, \Psi_{i} y_{t}\right\rangle-\left\langle y_{t}-u_{n_{j}, i}, \Psi_{i} x_{n_{j}}\right\rangle \\
& -\left\langle y_{t}-u_{n_{j}, i}, \frac{u_{n_{j}, i}-x_{n_{j}}}{r_{n_{j}}}\right\rangle+G_{i}\left(y_{t}, u_{n_{j}, i}\right) \\
= & \left\langle y_{t}-u_{n_{j}, i}, \Psi_{i} y_{t}-\Psi_{i} u_{n_{j}, i}\right\rangle+\left\langle y_{t}-u_{n_{j}, i}, \Psi_{i} u_{n_{j}, i}-\Psi_{i} x_{n_{j}}\right\rangle \\
& -\left\langle y_{t}-u_{n_{j}, i}, \frac{u_{n_{j}, i}-x_{n_{j}}}{r_{n_{j}}}\right\rangle+G_{i}\left(y_{t}, u_{n_{j}, i}\right) .
\end{aligned}
$$

Since $\left\|u_{n_{j}, i}-x_{n_{j}}\right\| \rightarrow 0$ as $j \rightarrow \infty$, we obtain that $\left\|\Psi_{i} u_{n_{j}, i}-\Psi_{i} x_{n_{j}}\right\| \rightarrow 0$ as $j \rightarrow \infty$. Furthermore, by the monotonicity of $\Psi_{i}$, we obtain that

$$
\left\langle y_{t}-u_{n_{j}, i}, \Psi_{i} y_{t}-\Psi_{i} u_{n_{j}, i}\right\rangle \geq 0
$$

Taking $j \rightarrow \infty$ in (3.41), we have from (A4) that

$$
\left\langle y_{t}-v, \Psi_{i} y_{t}\right\rangle \geq G_{i}\left(y_{t}, v\right) .
$$

Now, from (A1), (A4), and (3.43), we also have

$$
\begin{aligned}
0 & =G_{i}\left(y_{t}, y_{t}\right) \leq t G_{i}\left(y_{t}, y\right)+(1-t) G_{i}\left(y_{t}, v\right) \\
& \leq t G_{i}\left(y_{t}, y\right)+(1-t)\left\langle y_{t}-v, \Psi_{i} y_{t}\right\rangle \\
& =t G_{i}\left(y_{t}, y\right)+(1-t) t\left\langle y-v, \Psi_{i} y_{t}\right\rangle
\end{aligned}
$$


which yields that

$$
G_{i}\left(y_{t}, y\right)+(1-t)\left\langle y-v, \Psi_{i} y_{t}\right\rangle \geq 0
$$

Taking $t \rightarrow 0$, we have, for each $y \in C$

$$
G_{i}(v, y)+\left\langle y-v, \Psi_{i} v\right\rangle \geq 0, \quad \forall i \in\{1,2, \ldots, k\}
$$

This shows $v \in \operatorname{GEP}\left(G_{i}, \Psi_{i}\right)$, for all $i=1,2, \ldots, k$. Then, $v \in \cap_{i=1}^{k} \operatorname{GEP}\left(G_{i}, \Psi_{i}\right)$. Hence we have $v \in F\left(T_{1}\right) \cap F\left(T_{2}\right) \cap\left(\cap_{i=1}^{k} \operatorname{GEP}\left(G_{i}, \Psi_{i}\right)\right):=\Omega$. So, we have from (3.36) that

$$
\limsup _{n \rightarrow \infty}\left\langle u-x^{*}, \omega_{n}-x^{*}\right\rangle=\left\langle u-x^{*}, v-x^{*}\right\rangle \leq 0
$$

By (C1), (C4), (3.15), (3.30), (3.47), and Lemma 2.5, we obtain that $\lim _{n \rightarrow \infty}\left\|\omega_{n}-x^{*}\right\|=0$. Hence we have from (3.29) that $\left\{x_{n}\right\}$ converges to $x^{*}$, where $x^{*}=P_{\Omega} u$.

Case B. Assume that there exists a subsequence $\left\{\Gamma_{n_{i}}\right\}_{i \geq 0}$ of $\left\{\Gamma_{n}\right\}_{n \geq 0}$ such that $\Gamma_{n_{i}}<\Gamma_{n_{i}+1}$ for all $i \in \mathbb{N}$. In this case, it follows from Lemma 2.4 that there exists a subsequence $\left\{\Gamma_{\tau(n)}\right\}$ of $\left\{\Gamma_{n}\right\}$ such that $\Gamma_{\tau(n)+1}>\Gamma_{\tau(n)}$, where $\tau: \mathbb{N} \rightarrow \mathbb{N}$ is defined by

$$
\tau(n)=\max \left\{k \leq n: \Gamma_{k}<\Gamma_{k+1}\right\}, \quad \forall n \in \mathbb{N} .
$$

So, from (3.12), that

$$
\left\|\omega_{\tau(n)+1}-x^{*}\right\|^{2}-\left\|\omega_{\tau(n)}-x^{*}\right\|^{2}+\gamma_{\tau(n)}\left(1-\gamma_{\tau(n)}\right)\left\|\omega_{\tau(n)}-T_{1} \omega_{\tau(n)}\right\|^{2} \leq \alpha_{\tau(n)}\left\|u_{\tau(n)}-x^{*}\right\|^{2}
$$

Since $\left\|\omega_{\tau(n)}-x^{*}\right\|^{2}:=\Gamma_{\tau(n)}<\Gamma_{\tau(n)+1}:=\left\|\omega_{\tau(n)+1}-x^{*}\right\|^{2}$, we have

$$
\gamma_{\tau(n)}\left(1-\gamma_{\tau(n)}\right)\left\|\omega_{\tau(n)}-T_{1} \omega_{\tau(n)}\right\|^{2} \leq \alpha_{\tau(n)}\left\|u_{\tau(n)}-x^{*}\right\|^{2}
$$

By (C1) and (C3), we have

$$
\lim _{n \rightarrow \infty}\left\|\omega_{\tau(n)}-T_{1} \omega_{\tau(n)}\right\|=0
$$

By (3.15), we have

$$
\left\|\omega_{\tau(n)+1}-x^{*}\right\|^{2} \leq\left(1-\alpha_{\tau(n)}\right)\left\|\omega_{\tau(n)}-x^{*}\right\|^{2}+2 \alpha_{\tau(n)}\left\langle u_{\tau(n)}-x^{*}, x_{\tau(n)+1}-x^{*}\right\rangle .
$$


Now, in view of $\Gamma_{\tau(n)}<\Gamma_{\tau(n)+1}$, we see that

$$
\begin{aligned}
\left\|\omega_{\tau(n)}-x^{*}\right\|^{2} \leq & 2\left\langle u_{\tau(n)}-x^{*}, x_{\tau(n)+1}-x^{*}\right\rangle \\
= & 2\left\langle u_{\tau(n)}-u, x_{\tau(n)+1}-x^{*}\right\rangle+2\left\langle u-x^{*}, x_{\tau(n)+1}-\omega_{\tau(n)}\right\rangle \\
& +2\left\langle u-x^{*}, \omega_{\tau(n)}-x^{*}\right\rangle .
\end{aligned}
$$

Furthermore, we also have from (3.13) that

$$
\begin{aligned}
& \beta_{\tau(n)}\left(1-\beta_{\tau(n)}\right)\left\|y_{\tau(n)}-T_{2} \omega_{\tau(n)}\right\|^{2} \leq \alpha_{\tau(n)}\left\|u_{\tau(n)}-x^{*}\right\|^{2}+\left\|\omega_{\tau(n)}-x^{*}\right\|^{2} \\
& -\left\|\omega_{\tau(n)+1}-x^{*}\right\|^{2} \\
& \leq \alpha_{\tau(n)}\left\|u_{\tau(n)}-x^{*}\right\|^{2} \text {. }
\end{aligned}
$$

Applying (C1) and (C2) to the last inequality, we get that

$$
\lim _{n \rightarrow \infty}\left\|y_{\tau(n)}-T_{2} \omega_{\tau(n)}\right\|=0
$$

By (C1), (3.16), (3.51), and (3.55), we have

$$
\lim _{n \rightarrow \infty}\left\|x_{\tau(n)+1}-\omega_{\tau(n)}\right\|=0 .
$$

By (3.33), we have

$$
\lim _{n \rightarrow \infty}\left\|\omega_{\tau(n)+1}-x_{\tau(n)+1}\right\|=0 .
$$

It follows from (3.56) and (3.57) that

$$
\lim _{n \rightarrow \infty}\left\|\omega_{\tau(n)+1}-\omega_{\tau(n)}\right\|=0
$$

Since $\left\{\omega_{\tau(n)}\right\}$ is a bounded sequence, there exists a subsequence $\left\{\omega_{\tau\left(n_{j}\right)}\right\}$ such that

$$
\limsup _{n \rightarrow \infty}\left\langle u-x^{*}, \omega_{\tau(n)}-x^{*}\right\rangle=\lim _{j \rightarrow \infty}\left\langle u-x^{*}, \omega_{\tau\left(n_{j}\right)}-x^{*}\right\rangle
$$

Following the same argument as the proof of Case A for $\left\{\omega_{\tau\left(n_{j}\right)}\right\}$, we have that

$$
\limsup _{n \rightarrow \infty}\left\langle u-x^{*}, \quad \omega_{\tau(n)}-x^{*}\right\rangle \leq 0
$$

Using (C4), (3.53), (3.56), and (3.60), we have that

$$
\lim _{n \rightarrow \infty}\left\|\omega_{\tau(n)}-x^{*}\right\|=0
$$


By (3.58) and (3.61), we have that

$$
\lim _{n \rightarrow \infty}\left\|\omega_{\tau(n)+1}-x^{*}\right\|=0
$$

By Lemma 2.4 (ii), we get $\lim _{n \rightarrow \infty} \Gamma_{n}=0$; that is $\lim _{n \rightarrow \infty}\left\|\omega_{n}-x^{*}\right\|=0$. We observe that

$$
\left\|x_{n+1}-x^{*}\right\|^{2} \leq \alpha_{n}\left\|u_{n}-x^{*}\right\|^{2}+\left(1-\alpha_{n}\right)\left\|\omega_{n}-x^{*}\right\|^{2}
$$

Applying (C1), (C4), and $\lim _{n \rightarrow \infty}\left\|\omega_{n}-x^{*}\right\|^{2}=0$, we have immediately

$$
\lim _{n \rightarrow \infty}\left\|x_{n}-x^{*}\right\|=0
$$

that is, $\left\{x_{n}\right\}$ converges strongly to $x^{*}$, where $x^{*}=P_{\Omega} u$. This completes the proof.

Setting $\Psi_{i} \equiv 0$ for all $i=1,2, \ldots, k$ in Theorem 3.1, we obtain the following result.

Corollary 3.2. Let $C$ be a nonempty closed convex subset of a Hilbert space $H$. For each $i=1,2, \ldots, k$, let $G_{i}: C \times C \rightarrow \mathbb{R}$ be a bifunction satisfying (A1)-(A4). For each $j=1,2$, let $T_{j}: C \rightarrow H$ be two quasi-nonexpansive mappings such that $I-T_{j}$ are demiclosed at zero with $\Omega:=F\left(T_{1}\right) \cap F\left(T_{2}\right) \cap$ $\left(\cap_{i=1}^{k} E P\left(G_{i}\right)\right) \neq \emptyset$. Let the sequences $\left\{x_{n}\right\},\left\{y_{n}\right\}$, and $\left\{z_{n}\right\}$ be defined by

$$
\begin{gathered}
x_{1} \in H, \\
G_{1}\left(u_{n, 1}, y\right)+\frac{1}{r_{n}}\left\langle y-u_{n, 1}, u_{n, 1}-x_{n}\right\rangle \geq 0, \quad \forall y \in C, \\
G_{2}\left(u_{n, 2}, y\right)+\frac{1}{r_{n}}\left\langle y-u_{n, 2}, u_{n, 2}-x_{n}\right\rangle \geq 0, \quad \forall y \in C, \\
\vdots \\
G_{k}\left(u_{n, k}, y\right)+\frac{1}{r_{n}}\left\langle y-u_{n, k}, u_{n, k}-x_{n}\right\rangle \geq 0, \quad \forall y \in C, \\
\omega_{n}=\frac{1}{k} \sum_{i=1}^{k} u_{n, i}, \\
y_{n}=\gamma_{n} \omega_{n}+\left(1-\gamma_{n}\right) T_{1} \omega_{n}, \\
z_{n}=\beta_{n} y_{n}+\left(1-\beta_{n}\right) T_{2} \omega_{n},
\end{gathered}
$$

where $\left\{\alpha_{n}\right\},\left\{\beta_{n}\right\},\left\{\gamma_{n}\right\}$ satisfy the following conditions.

(C1) $\lim _{n \rightarrow \infty} \alpha_{n}=0$ and $\sum_{n=1}^{\infty} \alpha_{n}=\infty$;

(C2) $\liminf _{n \rightarrow \infty} \beta_{n}\left(1-\beta_{n}\right)>0$;

(C3) $\liminf _{n \rightarrow \infty} \gamma_{n}\left(1-\gamma_{n}\right)>0$;

(C4) $\lim _{n \rightarrow \infty} u_{n}=u$ for some $u \in H$. 
Then $\left\{x_{n}\right\}$ converges strongly to $x^{*}$, where $x^{*}=P_{\Omega} u$.

In the next results, using Theorem 3.1, we have new strong convergence theorems for two nonexpansive mappings in a Hilbert space.

Corollary 3.3. Let $C$ be a nonempty closed convex subset of a Hilbert space $H$. For each $i=1,2, \ldots, k$, let $G_{i}: C \times C \rightarrow \mathbb{R}$ be a bifunction satisfying (A1)-(A4) and $\Psi_{i}$ a $\mu_{i}$-inverse strongly monotone mapping. For each $j=1,2$, let $T_{j}: C \rightarrow H$ be two nonexpansive mappings such that $\Omega:=F\left(T_{1}\right) \cap$ $F\left(T_{2}\right) \cap\left(\cap_{i=1}^{k} \operatorname{GEP}\left(G_{i}, \Psi_{i}\right)\right) \neq \emptyset$. Let the sequences $\left\{x_{n}\right\},\left\{y_{n}\right\}$, and $\left\{z_{n}\right\}$ be defined by (3.1), where $\left\{\alpha_{n}\right\},\left\{\beta_{n}\right\},\left\{\gamma_{n}\right\}$ satisfy the following conditions.

(C1) $\lim _{n \rightarrow \infty} \alpha_{n}=0$ and $\sum_{n=1}^{\infty} \alpha_{n}=\infty$;

(C2) $\liminf _{n \rightarrow \infty} \beta_{n}\left(1-\beta_{n}\right)>0$;

(C3) $\liminf _{n \rightarrow \infty} \gamma_{n}\left(1-\gamma_{n}\right)>0$;

(C4) $\lim _{n \rightarrow \infty} u_{n}=u$ for some $u \in H$.

Then $\left\{x_{n}\right\}$ converges strongly to $x^{*}$, where $x^{*}=P_{\Omega} u$.

\section{Applications}

In this section, we present some convergence theorems deduced from the results in the previous section. Recall that a mapping $T: C \rightarrow H$ is said to be nonspreading [2] if

$$
2\|T x-T y\|^{2} \leq\|T x-y\|^{2}+\|T y-x\|^{2}
$$

for all $x, y \in C$. Further, a mapping $T: C \rightarrow H$ is said to be hybrid [23] if

$$
3\|T x-T y\|^{2} \leq\|x-y\|^{2}+\|T x-y\|^{2}+\|T y-x\|^{2}
$$

for all $x, y \in C$. These mappings are deduced from a firmly nonexpansive mapping in a Hilbert space.

A mapping $F: C \rightarrow H$ is said to be firmly nonexpansive if

$$
\|F x-F y\|^{2} \leq\langle x-y, F x-F y\rangle
$$

for all $x, y \in C$; see, for instance, Browder [24] and Goebel and Kirk [25]. We also know that a firmly nonexpansive mapping $F$ can be deduced from an equilibrium problem in a Hilbert space.

Recently, Kocourek et al. [26] introduced a more broad class of nonlinear mappings call generalized hybrid if there are $\alpha, \beta \in \mathbb{R}$ such that

$$
\alpha\|T x-T y\|^{2}+(1-\alpha)\|x-T y\|^{2} \leq \beta\|T x-y\|^{2}+(1-\beta)\|x-y\|^{2}
$$


for all $x, y \in C$. Very recently, they defined a more broad class of mappings than the class of generalized hybrid mappings in a Hilbert space. A mapping $S: C \rightarrow H$ is called super hybrid if there are $\alpha, \beta, \gamma \in \mathbb{R}$ such that

$$
\begin{aligned}
\alpha\|S x-S y\|^{2}+(1-\alpha+\gamma)\|x-S y\|^{2} \leq & (\beta+(\beta-\alpha) \gamma)\|S x-y\|^{2}+(1-\beta-(\beta-\alpha-1) \gamma)\|x-y\|^{2} \\
& +(\alpha-\beta) \gamma\|x-S x\|^{2}+\gamma\|y-S y\|^{2}
\end{aligned}
$$

for all $x, y \in C$. We call such a mapping an $(\alpha, \beta, \gamma)$-super hybrid mapping. We notice that an $(\alpha, \beta, 0)$-super hybrid mapping is $(\alpha, \beta)$-generalized hybrid. So, the class of super hybrid mappings contains the class of generalized hybrid mappings. A super hybrid mapping is not quasi-nonexpansive generally. For more details, see [27]. Before proving, we need the following lemmas.

Lemma 4.1 (see [27]). Let $C$ be a nonempty subset of a Hilbert space $H$ and let $\alpha, \beta$ and $\gamma$ be real numbers with $\gamma \neq-1$. Let $S$ and $T$ be mappings of $C$ into $H$ such that $S=(1 /(1+\gamma)) T+(\gamma /(1+\gamma)) I$. Then, $T$ is $(\alpha, \beta, \gamma)$-super hybrid if and only if $S$ is $(\alpha, \beta)$-generalized hybrid. In this case, $F(S)=F(T)$.

Lemma 4.2 (see [27]). Let $H$ be a Hilbert space and let $C$ be a nonempty closed convex subset of $H$. Let $S: C \rightarrow H$ be a generalized hybrid mapping. Then $S$ is demiclosed on $C$.

Setting $S_{j}:=\left(1 /\left(1+\gamma_{j}\right)\right) T_{j}+\left(\gamma_{j} /\left(1+\gamma_{j}\right)\right)$ I in Theorem 3.1, where $T_{j}$ is a super hybrid mapping and $\gamma_{j}$ is a real number, we obtain the following result.

Theorem 4.3. Let $C$ be a nonempty closed convex subset of a Hilbert space $H$. For each $i=1,2, \ldots, k$, let $G_{i}: C \times C \rightarrow \mathbb{R}$ be a bifunction satisfying (A1)-(A4) and $\Psi_{i}$ a $\mu_{i}$-inverse strongly monotone mapping. For each $j=1,2$, let $T_{j}: C \rightarrow H$ be $\left(\alpha_{j}, \beta_{j}, \gamma_{j}\right)$-super hybrid mappings such that $\Omega:=$ $F\left(T_{1}\right) \cap F\left(T_{2}\right) \cap\left(\cap_{i=1}^{k} \operatorname{GEP}\left(G_{i}, \Psi_{i}\right)\right) \neq \emptyset$. Let the sequences $\left\{x_{n}\right\},\left\{y_{n}\right\}$, and $\left\{z_{n}\right\}$ be defined by

$$
\begin{gathered}
x_{1} \in H, \\
G_{1}\left(u_{n, 1}, y\right)+\left\langle\Psi_{1} x_{n}, y-u_{n, 1}\right\rangle+\frac{1}{r_{n}}\left\langle y-u_{n, 1}, u_{n, 1}-x_{n}\right\rangle \geq 0, \quad \forall y \in C, \\
G_{2}\left(u_{n, 2}, y\right)+\left\langle\Psi_{2} x_{n}, y-u_{n, 2}\right\rangle+\frac{1}{r_{n}}\left\langle y-u_{n, 2}, u_{n, 2}-x_{n}\right\rangle \geq 0, \quad \forall y \in C, \\
\vdots \\
G_{k}\left(u_{n, k}, y\right)+\left\langle\Psi_{k} x_{n}, y-u_{n, k}\right\rangle+\frac{1}{r_{n}}\left\langle y-u_{n, k}, u_{n, k}-x_{n}\right\rangle \geq 0, \quad \forall y \in C, \\
\omega_{n}=\frac{1}{k} \sum_{i=1}^{k} u_{n, i}, \\
y_{n}=\gamma_{n} \omega_{n}+\left(1-\gamma_{n}\right)\left(\frac{1}{1+\gamma_{1}} T_{1} \omega_{n}+\frac{\gamma_{1}}{1+\gamma_{1}} \omega_{n}\right), \\
z_{n}=\beta_{n} y_{n}+\left(1-\beta_{n}\right)\left(\frac{1}{1+\gamma_{2}} T_{2} \omega_{n}+\frac{\gamma_{2}}{1+\gamma_{2}} \omega_{n}\right), \\
x_{n+1}=\alpha_{n} u_{n}+\left(1-\alpha_{n}\right) z_{n}, \quad \forall n \in \mathbb{N},
\end{gathered}
$$


where $\left\{\alpha_{n}\right\},\left\{\beta_{n}\right\},\left\{\gamma_{n}\right\}$ are sequences in $(0,1)$ and $\left\{u_{n}\right\} \subset H$ is a sequence and $\left\{r_{n}\right\} \subset\left[a, 2 \mu_{i}\right)$ for some $a>0$ and for all $i \in\{1,2, \ldots, k\}$. Suppose the following conditions are satisfied.

(C1) $\lim _{n \rightarrow \infty} \alpha_{n}=0$ and $\sum_{n=1}^{\infty} \alpha_{n}=\infty$;

(C2) $\liminf _{n \rightarrow \infty} \beta_{n}\left(1-\beta_{n}\right)>0$;

(C3) $\liminf _{n \rightarrow \infty} \gamma_{n}\left(1-\gamma_{n}\right)>0$;

(C4) $\lim _{n \rightarrow \infty} u_{n}=u$ for some $u \in H$.

Then $\left\{x_{n}\right\}$ converges strongly to $x^{*}$, where $x^{*}=P_{\Omega} u$.

Proof. For each $j=1,2$, setting

$$
S_{j}=\frac{1}{1+\gamma_{j}} T_{j}+\frac{\gamma_{j}}{1+\gamma_{j}} I
$$

we have from Lemma 4.1 that each $S_{j}$ is a generalized hybrid mapping and $F\left(S_{j}\right)=$ $F\left(T_{j}\right)$. Since $F\left(S_{j}\right) \neq \emptyset$, we have that each $S_{j}$ is quasi-nonexpansive. Following the proof of Theorem 3.1 and applying Lemma 4.2, we have the desired result. This completes the proof.

Setting $\Psi \equiv 0$ in Theorem 4.3, we obtains the following result.

Corollary 4.4. Let $C$ be a nonempty closed convex subset of a Hilbert space $H$. For each $i=1,2, \ldots, k$, let $G_{i}: C \times C \rightarrow \mathbb{R}$ be a bifunction satisfying (A1)-(A4). For each $j=1,2$, let $T_{j}: C \rightarrow H$ be $\left(\alpha_{j}, \beta_{j}, \gamma_{j}\right)$-super hybrid mappings such that $\Omega:=F\left(T_{1}\right) \cap F\left(T_{2}\right) \cap\left(\cap_{i=1}^{k} E P\left(G_{i}\right)\right) \neq \emptyset$. Let the sequences $\left\{x_{n}\right\},\left\{y_{n}\right\}$, and $\left\{z_{n}\right\}$ be defined by

$$
\begin{gathered}
x_{1} \in H, \\
G_{1}\left(u_{n, 1}, y\right)+\frac{1}{r_{n}}\left\langle y-u_{n, 1}, u_{n, 1}-x_{n}\right\rangle \geq 0, \quad \forall y \in C, \\
G_{2}\left(u_{n, 2}, y\right)+\frac{1}{r_{n}}\left\langle y-u_{n, 2}, u_{n, 2}-x_{n}\right\rangle \geq 0, \quad \forall y \in C, \\
\vdots \\
G_{k}\left(u_{n, k}, y\right)+\frac{1}{r_{n}}\left\langle y-u_{n, k}, u_{n, k}-x_{n}\right\rangle \geq 0, \quad \forall y \in C, \\
\omega_{n}=\frac{1}{k} \sum_{i=1}^{k} u_{n, i}, \\
y_{n}=\gamma_{n} \omega_{n}+\left(1-\gamma_{n}\right)\left(\frac{1}{1+\gamma_{1}} T_{1} \omega_{n}+\frac{\gamma_{1}}{1+\gamma_{1}} \omega_{n}\right), \\
z_{n}=\beta_{n} y_{n}+\left(1-\beta_{n}\right)\left(\frac{1}{1+\gamma_{2}} T_{2} \omega_{n}+\frac{\gamma_{2}}{1+\gamma_{2}} \omega_{n}\right), \\
x_{n+1}=\alpha_{n} u_{n}+\left(1-\alpha_{n}\right) z_{n}, \quad \forall n \in \mathbb{N},
\end{gathered}
$$


where $\left\{\alpha_{n}\right\},\left\{\beta_{n}\right\},\left\{\gamma_{n}\right\}$ are sequences in $(0,1)$ and $\left\{u_{n}\right\} \subset H$ is a sequence and $\left\{r_{n}\right\} \subset[a, \infty)$ for some $a>0$. Suppose the following conditions are satisfied.

(C1) $\lim _{n \rightarrow \infty} \alpha_{n}=0$ and $\sum_{n=1}^{\infty} \alpha_{n}=\infty$;

(C2) $\liminf _{n \rightarrow \infty} \beta_{n}\left(1-\beta_{n}\right)>0$;

(C3) $\liminf _{n \rightarrow \infty} \gamma_{n}\left(1-\gamma_{n}\right)>0$;

(C4) $\lim _{n \rightarrow \infty} u_{n}=u$ for some $u \in H$.

Then $\left\{x_{n}\right\}$ converges strongly to $x^{*}$, where $x^{*}=P_{\Omega} u$.

In Corollary 4.4, put $G_{i}(x, y)=0$ for all $x, y \in C$ and $r_{n}=1$ for all $n \in \mathbb{N}$. Then we have that $u_{n, i}=x_{n}$ for all $i=1,2, \ldots, k$, which gives that $\omega_{n}=(1 / k) \sum_{i=1}^{k} u_{n, i}=x_{n}$. Thus we obtain the following results from Corollary 4.4 .

Corollary 4.5. Let $C$ be a nonempty closed convex subset of a Hilbert space $H$. For each $j=1,2$, let $T_{j}: C \rightarrow H$ be $\left(\alpha_{j}, \beta_{j}, \gamma_{j}\right)$-super hybrid mappings such that $F\left(T_{1}\right) \cap F\left(T_{2}\right) \neq \emptyset$. Let the sequences $\left\{x_{n}\right\},\left\{y_{n}\right\}$, and $\left\{z_{n}\right\}$ be defined by

$$
\begin{gathered}
x_{1} \in H, \\
y_{n}=\gamma_{n} x_{n}+\left(1-\gamma_{n}\right)\left(\frac{1}{1+\gamma_{1}} T_{1} x_{n}+\frac{\gamma_{1}}{1+\gamma_{1}} x_{n}\right), \\
z_{n}=\beta_{n} y_{n}+\left(1-\beta_{n}\right)\left(\frac{1}{1+\gamma_{2}} T_{2} x_{n}+\frac{\gamma_{2}}{1+\gamma_{2}} x_{n}\right), \\
x_{n+1}=\alpha_{n} u_{n}+\left(1-\alpha_{n}\right) z_{n}, \quad \forall n \in \mathbb{N},
\end{gathered}
$$

where $\left\{\alpha_{n}\right\},\left\{\beta_{n}\right\},\left\{\gamma_{n}\right\}$ are sequences in $(0,1)$ and $\left\{u_{n}\right\} \subset H$ is a sequence. Suppose the following conditions are satisfied.

(C1) $\lim _{n \rightarrow \infty} \alpha_{n}=0$ and $\sum_{n=1}^{\infty} \alpha_{n}=\infty$;

(C2) $\liminf _{n \rightarrow \infty} \beta_{n}\left(1-\beta_{n}\right)>0$;

(C3) $\liminf _{n \rightarrow \infty} \gamma_{n}\left(1-\gamma_{n}\right)>0$;

(C4) $\lim _{n \rightarrow \infty} u_{n}=u$ for some $u \in H$.

Then $\left\{x_{n}\right\}$ converges strongly to $x^{*}$, where $x^{*}=P_{F\left(T_{1}\right) \cap F\left(T_{2}\right)} u$.

In Corollary 4.5, put $T_{1}=I$, the identity mapping, and $T_{2}:=T$, an $(\alpha, \beta, \gamma)$-super hybrid mapping. Thus we obtain the following results.

Corollary 4.6. Let $C$ be a nonempty closed convex subset of a Hilbert space $H$. Let $T$ be an $(\alpha, \beta, \gamma)$ super hybrid mapping such that $F(T) \neq \emptyset$. Let the sequences $\left\{x_{n}\right\},\left\{y_{n}\right\}$, and $\left\{z_{n}\right\}$ be defined by

$$
\begin{gathered}
x_{1} \in H, \\
z_{n}=\beta_{n} x_{n}+\left(1-\beta_{n}\right)\left(\frac{1}{1+\gamma} T x_{n}+\frac{\gamma}{1+\gamma} x_{n}\right), \\
x_{n+1}=\alpha_{n} u_{n}+\left(1-\alpha_{n}\right) z_{n}, \quad \forall n \in \mathbb{N},
\end{gathered}
$$


where $\left\{\alpha_{n}\right\}$ and $\left\{\beta_{n}\right\}$ are sequences in $(0,1)$ and $\left\{u_{n}\right\} \subset H$ is a sequence. Suppose the following conditions are satisfied.

(C1) $\lim _{n \rightarrow \infty} \alpha_{n}=0$ and $\sum_{n=1}^{\infty} \alpha_{n}=\infty$;

(C2) $\liminf _{n \rightarrow \infty} \beta_{n}\left(1-\beta_{n}\right)>0$;

(C3) $\lim _{n \rightarrow \infty} u_{n}=u$ for some $u \in H$.

Then $\left\{x_{n}\right\}$ converges strongly to $x^{*}$, where $x^{*}=P_{F(T)} u$.

\section{Acknowledgments}

The first author is supported by the National Research Council of Thailand. The authors would like to thank the referees for reading this paper carefully, providing valuable suggestions and comments, and pointing out a major error in the original version of this paper.

\section{References}

[1] S. Itoh and W. Takahashi, "The common fixed point theory of singlevalued mappings and multivalued mappings," Pacific Journal of Mathematics, vol. 79, no. 2, pp. 493-508, 1978.

[2] F. Kohsaka and W. Takahashi, "Fixed point theorems for a class of nonlinear mappings related to maximal monotone operators in Banach spaces," Archiv der Mathematik, vol. 91, no. 2, pp. 166-177, 2008.

[3] A. Moudafi, "Weak convergence theorems for nonexpansive mappings and equilibrium problems," Journal of Nonlinear and Convex Analysis, vol. 9, no. 1, pp. 37-43, 2008.

[4] E. Blum and W. Oettli, "From optimization and variational inequalities to equilibrium problems," The Mathematics Student, vol. 63, no. 1-4, pp. 123-146, 1994.

[5] P. L. Combettes and S. A. Hirstoaga, "Equilibrium programming in Hilbert spaces," Journal of Nonlinear and Convex Analysis, vol. 6, no. 1, pp. 117-136, 2005.

[6] S. Takahashi and W. Takahashi, "Viscosity approximation methods for equilibrium problems and fixed point problems in Hilbert spaces," Journal of Mathematical Analysis and Applications, vol. 331, no. 1, pp. 506-515, 2007.

[7] L. C. Ceng and J. C. Yao, "A relaxed extragradient-like method for a generalized mixed equilibrium problem, a general system of generalized equilibria and a fixed point problem," Nonlinear Analysis, vol. 72, no. 3-4, pp. 1922-1937, 2010.

[8] P.-E. Mainge and A. Moudafi, "Coupling viscosity methods with the extragradient algorithm for solving equilibrium problems," Journal of Nonlinear and Convex Analysis, vol. 9, no. 2, pp. 283-294, 2008.

[9] R. Wangkeeree, “An extragradient approximation method for equilibrium problems and fixed point problems of a countable family of nonexpansive mappings," Fixed Point Theory and Applications, vol. 2008, Article ID 134148, 2008.

[10] R. Wangkeeree and U. Kamraksa, "An iterative approximation method for solving a general system of variational inequality problems and mixed equilibrium problems," Nonlinear Analysis. Hybrid Systems, vol. 3, no. 4, pp. 615-630, 2009.

[11] R. Wittmann, "Approximation of fixed points of nonexpansive mappings," Archiv der Mathematik, vol. 58, no. 5, pp. 486-491, 1992.

[12] W. Takahashi, Convex Analysis and Approximation of Fixed Points, vol. 2, Yokohama Publishers, Yokohama, Japan, 2000.

[13] B. Halpern, "Fixed points of nonexpanding maps," Bulletin of the American Mathematical Society, vol. 73, pp. 957-961, 1967.

[14] Y. Kurokawa and W. Takahashi, "Weak and strong convergence theorems for nonspreading mappings in Hilbert spaces," Nonlinear Analysis, vol. 73, no. 6, pp. 1562-1568, 2010.

[15] M. Hojo and W. Takahashi, "Weak and strong convergence theorems for generalized hybrid mappings in Hilbert spaces," Scientiae Mathematicae Japonicae, vol. 73, no. 1, pp. 31-40, 2011. 
[16] Y. Yao and N. Shahzad, "New methods with perturbations for non-expansive mappings in Hilbert spaces," Fixed Point Theory and Applications, vol. 2011, article 79, 2011.

[17] C. S. Chuang, L. J. Lin, and W. Takahashi, "Halpern's type iterations with perturbations in Hilbert spaces: equilibriumsolutions and fixed points," Journal of Global Optimization. In press.

[18] S. Takahashi and W. Takahashi, "Strong convergence theorem for a generalized equilibrium problem and a nonexpansive mapping in a Hilbert space," Nonlinear Analysis, vol. 69, no. 3, pp. 1025-1033, 2008.

[19] W. Takahashi, Introduction to Nonlinear and Convex Analysis, Yokohama Publishers, Yokohama, Japan, 2009.

[20] W. Takahashi, Nonlinear Functional Analysis-Fixed Point Theory and Its Applications, Yokohama Publishers, Yokohama, Japan, 2000.

[21] P. E. Mainge, "Strong convergence of projected subgradient methods for nonsmooth and nonstrictly convex minimization," Set-Valued Analysis, vol. 16, no. 7-8, pp. 899-912, 2008.

[22] K. Aoyama, Y. Kimura, W. Takahashi, and M. Toyoda, "Approximation of common fixed points of a countable family of nonexpansive mappings in a Banach space," Nonlinear Analysis, vol. 67, no. 8, pp. 2350-2360, 2007.

[23] W. Takahashi, "Fixed point theorems for new nonlinear mappings in a Hilbert space," Journal of Nonlinear and Convex Analysis, vol. 11, no. 1, pp. 79-88, 2010.

[24] F. E. Browder, "Convergence theorems for sequences of nonlinear operators in Banach spaces," Mathematische Zeitschrift, vol. 100, pp. 201-225, 1967.

[25] K. Goebel and W. A. Kirk, Topics in Metric Fixed Point Theory, vol. 28 of Cambridge Studies in Advanced Mathematics, Cambridge University Press, Cambridge, UK, 1990.

[26] P. Kocourek, W. Takahashi, and J. C. Yao, "Fixed point theorems and weak convergence theorems for generalized hybrid mappings in Hilbert spaces," Taiwanese Journal of Mathematics, vol. 14, no. 6, pp. 2497-2511, 2010.

[27] W. Takahashi, J. C. Yao, and P. Kocourek, "Weak and strong convergence theorems for generalized hybrid nonself-mappings in Hilbert spaces," Journal of Nonlinear and Convex Analysis, vol. 11, no. 3, pp. 567-586, 2010. 


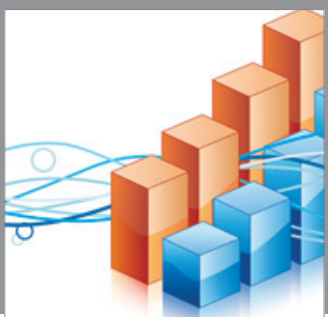

Advances in

Operations Research

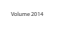

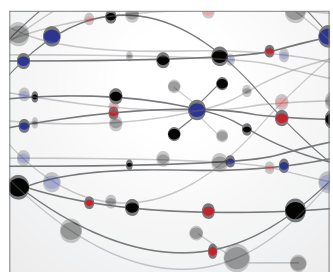

\section{The Scientific} World Journal
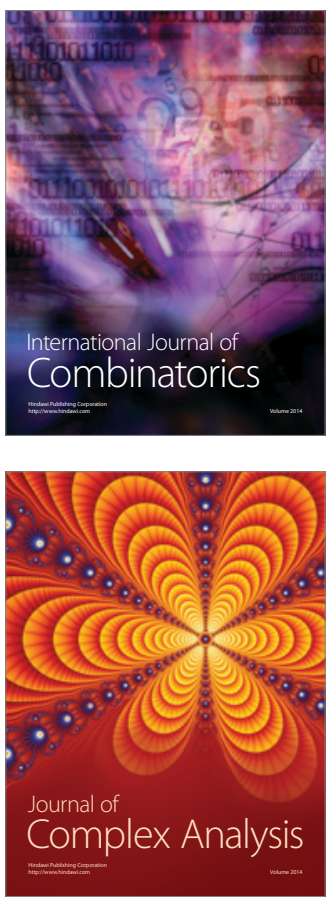

International Journal of

Mathematics and

Mathematical

Sciences
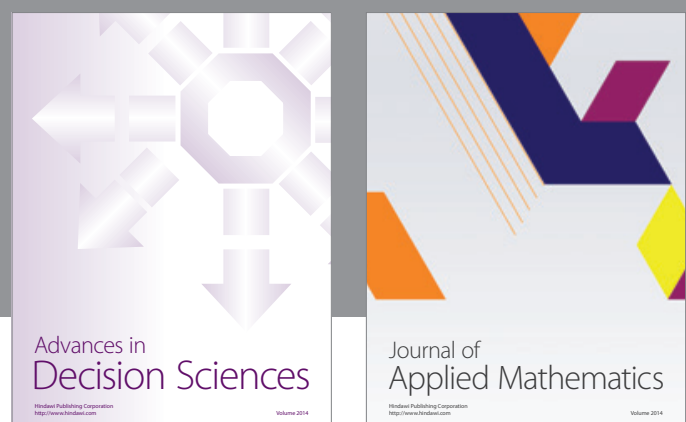

Journal of

Applied Mathematics
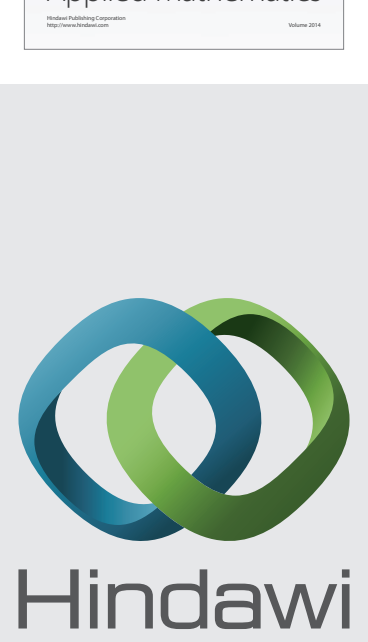

Submit your manuscripts at http://www.hindawi.com
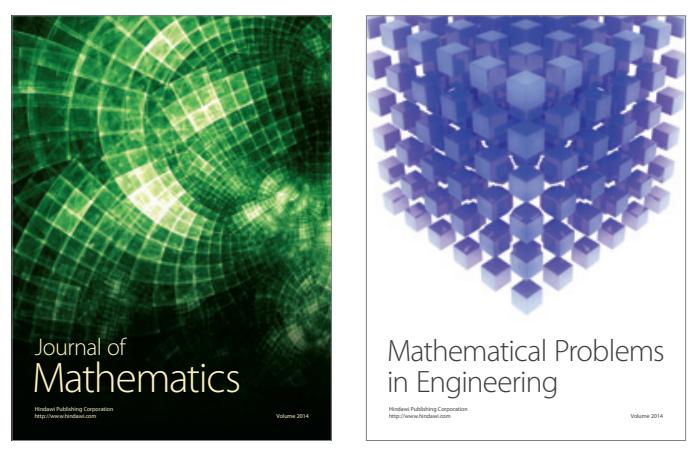

Mathematical Problems in Engineering
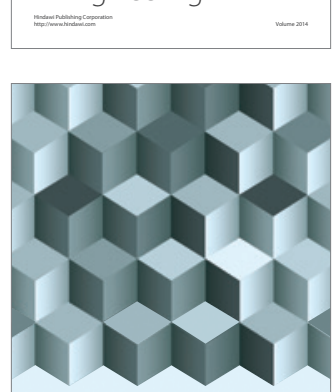

Journal of

Function Spaces
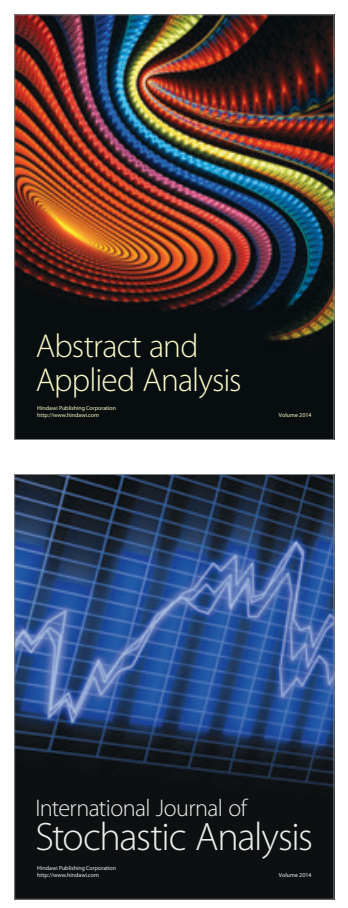

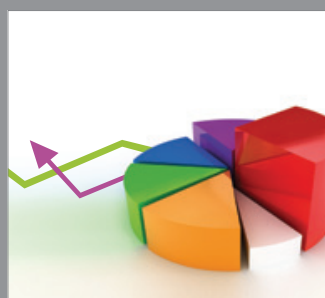

ournal of

Probability and Statistics

Promensencen
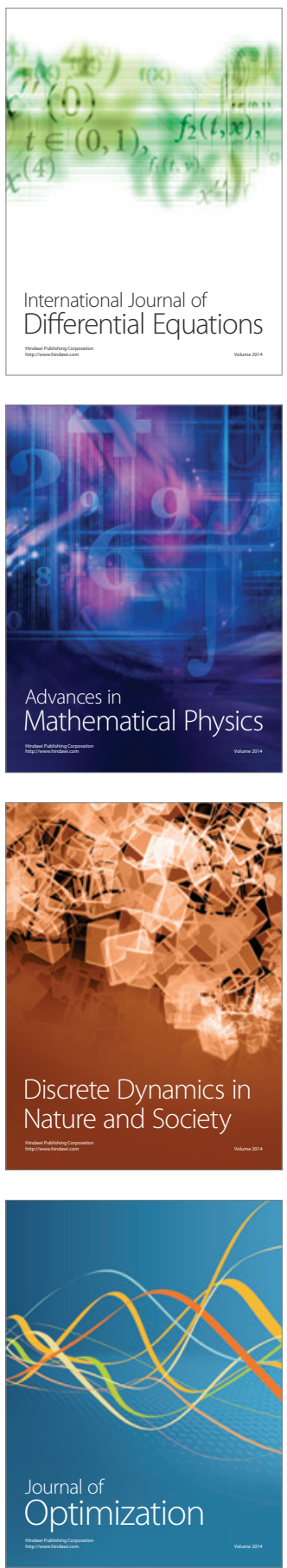